\title{
The Role of General Collective Intelligence in Optimizing Future Educational Environments
}

\author{
Andy E. Williams ${ }^{1, *}$ \\ ${ }^{1}$ Nobeah Foundation, Nairobi, Kenya
}

\begin{abstract}
From a functional perspective, an analysis of an educational environment serves a useful function to the degree it can reliably improve educational outcomes. However, both the choice of outcomes to optimize, and the choice of how to measure impact of educational initiatives on that optimization, might often be both subjective. General Collective Intelligence or GCI is a group decision-making system with the potential to significantly increase impact on any general outcome through increasing a group's general problem-solving ability (intelligence). Having general problem-solving ability, a GCI must have the capacity to not only choose the optimal solution to problems, but also to choose the optimal problem to solve. However, while the subject of education has driven seeming endless analysis, the education of a nation's children might often be too sensitive and too subjective of a topic for discussion to converge on consensus regarding such fundamental issues. As a result, without the ability to agree on what the goals (targeted outcomes) of education should even be, the problem of optimizing educational outcomes might not be susceptible to any analysis with the capacity to reliably achieve a significant improvement in such outcomes. However, with functional modeling this is poised to change. This paper explores how the properties of educational environments might be represented with functional modeling so that a General Collective Intelligence (GCI) might reliably optimize educational outcomes to a far greater degree than possible today.
\end{abstract}

Keywords: General Collective Intelligence, Human-Centric Functional Modelling, Artificial General Intelligence, education

\section{Background}

General Collective Intelligence or GCI [1], [2] has been defined as a platform that organizes groups into a single collective cognition with the potential for vastly greater general problem solving ability than any individual in the group. Where outcomes are constrained by general problem-solving ability, as opposed to being constrained by any physical limits, GCI has the potential to vastly increase outcomes in comparison to any other possible intervention. In order to apply the collective reasoning of a GCI however, information and processes must be represented in a common way for the entire group. Human-
Centric Functional Modeling (HCFM) [3] provides this common mechanism.

In addition to vastly increasing outcome to a degree not reliably achievable otherwise, implementation of GCI itself is a large complex initiative that needs to be spread across a large number of educational and other project in order for the required expenditure per project to be small enough in relation to the project budget to be feasible. In order to deploy a GCI based educational or other program, entire networks of cooperating projects [4] are required to deploy GCI functionality in a hierarchy according to its dependencies. Without massive top-down support,

"Corresponding author. Email: awilliams@nobeahfoundation.org 
such networks of cooperation might need to be created by designing processes that self-assemble in ways that create the necessary resources and capacity. That is, by designing processes that begin with the minimal set of GCI functionality that can be implemented by an individual or small team, then use that functionality to increase impact on some educational outcome while engaging more people and resources to build more GCI functionality, then using that GCI functionality to achieve increased educational outcomes and to engage more people and resources to build yet more GCI functionality, in an iterative cycle in order to evolve an implementation of GCI and an implementation of a GCI based educational solution. Because these components are evolved rather than designed in a top-down process, the actual components and their final configuration cannot be completely determined beforehand.

\section{Introduction}

In the initial phase of a collective intelligence based educational program, the specific set of educational outcomes to be targeted must preselected rather than being selected by a GCI process. And a small subset of GCI functionality must be chosen to be implemented in order to increase impact on these targeted outcomes. The goal of implementing this small subset of GCI functionality is to introduce additional GCI functionality that reduces the barriers against considering a wider range of educational interventions, and to improve ability to more reliably select the educational interventions with the greatest fitness in functioning to improve each specific educational outcome.

In future iterations of such a program, as more and more GCI functionality is implemented, the goal of applying GCI to the improvement of educational outcomes will also become to more intelligently select the outcomes that should be targeted by education, that is, to select the educational outcomes with the greatest fitness in functioning to improve the dimensions of wellbeing that are related to education. GCI introduces a universal scale of measurement so that such a determination is even possible. Because without a universal understanding of what constitutes an excellent education, perhaps some believing it means an encyclopedic knowledge of facts, and others believing it means a basic set of cognitive tools with which it is possible to navigate a wide range of facts that can be looked up, there might not be any possibility of collectively converging on agreement as to the ideal interventions to achieve any outcome whatsoever.

\section{Related Work}

Though others have described the concept of a general collective intelligence factor (c factor) [5], though at least one other has defined a model for a general collective intelligence factor [6], and though others have described the concept of a collective super intelligence [7], to the author's knowledge the author's own work is the only model that defines the specific mechanisms required for a General Collective Intelligence or GCI with the potential for exponentially greater general problem-solving ability (intelligence) than any individual [1]. Because of this, and because this model is so new, all of the existing literature surrounding this GCI refers to the author's own published work, or pre-prints of the author's unpublished work that is still under review.

\section{Purpose, Research Questions, and Approach}

The purpose of this paper was to demonstrate the capacity of General Collective Intelligence to significantly increase any targeted educational outcomes through individualizing educational services to increase the three capacities through which General Collective Intelligence might significantly increase any targeted outcome [2]. And to demonstrate the capacity of General Collective Intelligence to target the right educational outcomes in order to maximize collective well-being through maximizing the well-being of every individual.

The research question addressed in this paper and the method with which it was approached are described below: 
Section 4: Research Question

4.1. Can General Collective Intelligence significantly increase any targeted educational outcomes?

\section{Section 5: Model}

5.1. Defining and measuring educational outcomes.

\section{Section 6. Method}

6.1. An analysis is performed to assess how functional models of educational interventions might be individualized.

6.2. An analysis is performed to assess whether and how the truth of conclusions driving individualized interventions might be determined.

6.3. An analysis is performed to assess whether interventions might be individually and collectively optimized according to such conclusions.

Section 7. Findings

Section 8. Research Limitations

Section 9. Practical Implications

Section 10. Conclusions

\section{Model}

Aspects of the functional modeling approach, and aspects of General Collective Intelligence that are relevant to the research questions are described below.

\subsection{Defining and Measuring Educational Outcomes to be Targeted}

GCI leverages Human-Centric Functional Modeling in order to individualize educational and other services [8], so that individual outcomes can be maximized in order to in turn maximize collective outcomes.

In the Human-Centric Functional Modeling approach, any system that can be observed (including the human organism itself, as well as the educational system) can be represented in terms of the basic functions by which it changes its state. These states form a functional state space through which the system moves. In the case of human-beings, any reasoning a human is capable of can be represented by a path through the "conceptual space" that acts as the functional state space of the cognitive system. Any problem encountered by a student, such as the problem of achieving an educational outcome, represents the lack of a path from the concept representing the initial state, to the concept representing the coal. In order to maximize attainment of that individual student's goal, the educational system must determine the individual student's intended path through their conceptual space, and the educational system must provide the optimal intervention to achieve that goal.

In the functional modeling approach required by GCI, the volume of any outcomes that is considered to have been achieved is defined in a common way for every process. An attempt has been made to track outcomes through a common methodology (the Discrete Distributed Work Management Methodology or DDWMM) [9], where a platform that automates this DDWMM is intended to facilitate management of an education program in a proposed Collective Intelligence based Program to Accelerate Achievement of the Sustainable Development Goals (CIPAA-SDGs) [10].

In this functional modeling approach the fitness of an intervention in functioning to achieve outcomes is determined by its relative volume of outcomes per volume of inputs in comparison to other interventions. Here "volume" is an abstract quantity that might be calculated based a common set of variables (e.g. Task Execution Speed, Task Execution Quality, Task Execution Reliability, Task Execution Difficulty, Task Estimation Accuracy) in order to measure outcomes achieved by teachers or to measure outcomes achieved by teaching tools:

Volume of Outcomes in Individual or Group Task Execution $=\mathrm{F}$ (Task Execution Speed, Task Execution Quality, Task Execution Reliability, Task Execution Difficulty, Task Estimation Accuracy) 
Every component of a GCI is intended to have the capacity to adapt to be replaced by another implementation that performs better. As a result, this function might be applied to itself or other functions that measure outcomes, so that the most accurate function can be selected.

In measuring the volume of outcomes achieved by a manager in managing the execution of tasks, such as through the role of principal, in one approach that volume is given by their impact on the volume of outcomes achieved by each team member they manage:

Volume of Outcomes in Management of Task Execution $=\mathrm{F}$ (Volume of Outcomes in Task Execution for Individuals in Team)

In measuring the volume of outcomes achieved in leading the management of tasks, such as through the role of superintendent, in one approach that volume is given by their impact on the volume of outcomes achieved by each management team member:

Volume of Outcomes in Leadership of Task Management $=\mathrm{F}$ (Volume of Outcomes in Task Execution Management for Managers in Team)

All metrics for subjective measures must be normalized to be between 0 and 1 so that all such metrics can be compared. That is: $0<$ Task Execution Speed, Task Execution Quality, Task Execution Reliability, Task Execution Difficulty, Task Estimation Accuracy $<1$.

In this functional model, well-being is the fitness to perform all functions within a given domain. The educational well-being of an individual might then be defined as their fitness to perform all the functions required by the processes of the current educational system with the existing outcomes that it targets. The outcomes targeted by a particular educational system might also be changed to better optimize the overall well-being of individual students. The overall or conscious well-being of an individual is defined as their fitness to perform all of the functions of their body, their emotions, their cognition, and their consciousness. The cognitive understanding of this well-being is a set of paths through conceptual space that might be optimized for each individual [8].

\section{Method}

The research question of whether GCI might significantly increase educational outcomes was addressed using the methods summarized in table 2.

Table 2. Research question and method.

\begin{tabular}{|c|c|}
\hline Research Question & Method \\
\hline 1. General & 1. Assess the \\
\hline Collective & requirements of \\
\hline Intelligence & General Collective \\
\hline significantly & Intelligence based \\
\hline increase any targeted & development of \\
\hline educational & education. \\
\hline outcomes? & 2. Assess whether \\
\hline & they might be \\
\hline & implemention of \\
\hline
\end{tabular}

\subsection{General Collective Intelligence Based Education Solution Development}

The general pattern by which GCI solutions reliably might achieve a significant increase in impact on any targeted outcome begins with defining the processes by which the outcomes are achieved, and then defining a semantic model capable of representing any process, and potentially defining a functional model (a semantic model limited to a given functional domain) of that process. This functional model might then be used by the software or other tools that might automate that process. The next step in education solution development is using GCI as an adaptive general problem-solving system to apply specific development problem-solving processes to increase the three critical capacities by which the ability of GCI to impact any targeted 
outcome can be increased. These increases in capacities are increased capacity for complexity, increased capacity to execute more processes, and increased capacity to align cooperation so that it creates sufficient value to be self-sustaining. Increasing these capacities for a given educational process, for a given educational tool, or for any other educational intervention, can then be used to increase educational outcomes.

Therefore, wherever outcomes in the educational domain can be increased through these three capacities without physical limits, GCI has the potential to exponentially increase educational outcomes. In the case that such physical limits do exist, GCI might still significantly increase such outcomes. Consider all processes involved in education, from the business processes of schools, to the business processes of services providers to schools, as well as others. Representing each process using a swim lane diagram, then the activities conducted by each participant in the process will be confined to each lane.

\section{Outcomes of Collective Processes}

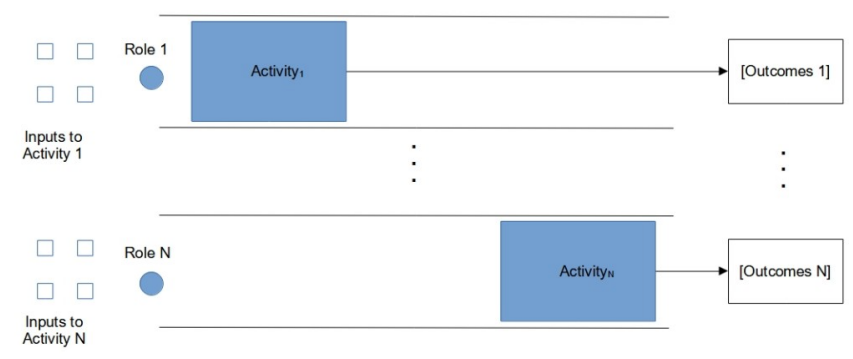

Figure 1. Swim lane diagram representing activities and participants in any process related to health or wellness care.

One way efficiency in achieving outcomes might be increased is to scale those processes over more, fewer, or potentially different participants wherever doing so makes processing more efficient. This includes automating some processes so they might be performed at greater speed and scale by fewer participants with more processing resources, or so they might be performed at greater speed and scale by more participants with less processing resources.
Having general problem-solving ability, a GCI as an adaptive collective problem-solving system might be applied to adapt such processes in this way.

General Collective Intelligence is defined within a Functional Modeling Framework (FMF) [11] that represents living organisms as consisting of a set of adaptive problem-solving processes that fall into a hierarchy of domains. One of these domains is cognition, but all of these domains are represented by processes that solve problems in that domain by keeping fitness to execute functions within the domain in a bounded region, so that the existence of such functions is stable. Since a problem in a functional state space is the lack of a path between a point representing one state, and a point representing another state, then general problem-solving ability in functional state space is the volume of space that can be potentially navigated per unit time in order to find a path between those two points (i.e. to find a solution to the problem). Since all problems in GCI are essentially solved by replicating the solution that life has developed and proved over billions of years, every problem solving processes is essentially a matter of determining how functional models of living processes can be abstracted in order to apply those solutions to more general problems.

In the case of education, the process of defining the optimal education for an individual might be functionally equivalent with the process of cells differentiating in the process of taking their final form. Cells differentiate in ways that are determined by internal factors (the cell's genome), as well as by external factors (the environment around the cell). A cell inside the brain that develops into a muscle cell might have poor outcomes in that it might never be allowed to function. Similarly, a cell on the palm of the hand that develops into a neuron might never be allowed to function either. In either case, differentiation must occur through some adaptive problem-solving process that reliably converges on the optimal function. And in order to reliably converge on the optimal function, that process 
must be aware of function, and therefore must model such functions.

The particular choice of educational outcome that is targeted by such a GCI based educational solution development program is then less important than targeting that outcome through a combination of functional modeling and GCI as a functional modeling based adaptive problemsolving process.

\subsection{General Collective Intelligence Based \\ Education Program Implementation}

A General Collective Intelligence based Education Program must define a self-assembling process to iteratively engage sufficient people and resources to deploy education solutions in a self-supporting network of cooperation with other projects [4]. Deployment in a self-supporting network might allow education solutions to be deployed at a scale at which sufficient impact to justify the education program might be achieved. This involves defining and deploying one or more selfsupporting educational value chains that incorporate them. The exact value chain might be self-selected by participating stakeholders during the self-assembling implementation.

One example is Education Solution Development in the proposed Collective Intelligence Based Program to Accelerate Achievement of the Sustainable Development Goals (CIPAA-SDGs). This educational program includes development and deployment of two educational solutions beginning in some pilot country, where those solutions leverage the principles of collective intelligence to more efficiently orchestrate cooperation between education providers, activities providers, students, parents groups, and others. Through this cooperation, without changing the goals that education systems are targeting at this point, these solutions are projected to create the capacity to achieve significant educational savings in the deployment region, or a significant increase in the services that can be delivered at the same cost, and thereby to increase access to affordable education across the region.

\section{Findings}

Through very high level analysis, the potential of General Collective Intelligence to significantly improve educational outcomes appeared to be confirmed.

Table 3. Research Findings.

\begin{tabular}{ll}
\hline Research Question & Findings \\
\hline 1. General Collective & The answer \\
Intelligence & appears to be yes \\
significantly increase & pending \\
any targeted & confirmation by an \\
educational outcomes? & actual \\
& implementation. \\
\hline
\end{tabular}

\section{Research Limitations}

It is outside of the scope of this paper to provide the functional models of the human system that are required to achieve mass individualization of educations services. A major effort to elaborate this functional model is required in order to do so. All functional state spaces are expected to have some common abstract properties. As the model of the conceptual space of the cognitive system continues to be elaborated, this commonality should enable that effort to benefit the understanding of the functional state space for the human system, and all other functional states spaces as well.

\section{Practical Implications}

This educational program as defined for the CARICOM region proposes to develop two educational platforms (the Education Management Platform, and the Activities Management Platform). These platforms are proposed to be developed in the pilot country within the CARICOM region potentially Jamaica, Trinidad and Tobago, or Antigua and Barbuda. These solutions are projected to create the capacity to achieve significant educational savings in the CARICOM region, or a significant increase in the services that can be delivered at the same cost, 
and thereby to increase access to affordable education across the region.

Using collective intelligence to systematically build large-scale cooperation with the potential for transformative impact on access to affordable education might create the opportunity to leverage the projected cost savings across the entire CARICOM region to incentivize a far larger investment in developing these solutions than would be justified by the size of any single CARICOM country alone. Cooperation might extend to all aspects of such programs, even funding. If launching such a program in such developing countries with less well-funded governments such a program might be launched with private investment incentivized by either donor grants, or grants of tax credits, both of which might be provided through "social impact bonds" [10] to support the implementation process.

Impact capacity bonds are additional instruments that might support the development process. Impact capacity bonds commit other countries to reimburse the program's investment in solution development. This commitment is made to reimburse that investment with a portion of the savings the committing country actually achieves with the solution developed. In other words the second country is paying stakeholders in the pilot country to invest in creating the capacity for this impact on educational cost saving, in return for the second country getting far more impact on educational cost saving than they could have achieved without GCI based cooperation, and with far less risk. For example, if even one fifth of the potential $50 \%$ cost savings are reliably possible, an impact capacity bond from another CARICOM member state might commit the second country to reimburse this program with $5 \%$ of the savings they achieve by use of that solution. Over all of CARICOM this savings could amount to over 50 times the investment in development of these solutions than could be justified by the size of the educational market in the pilot country alone. Through this cooperation, this program aims to create solutions that address specific Educational challenges shared by all the islands, that aren't solvable within the resources of any island alone.

\subsection{Education Solution Development in the Collective Intelligence Based Program to Accelerate Achievement of the Sustainable Development Goals (CIPAA-SDGs)}

In the United States the Collective Intelligence Based Educational Program described in this summary aims to first apply GCI to homeschool related education processes in order to develop a solutions through which it is possible to provide access to more affordable education by cooperating with other local homeschool stakeholders to achieve $25 \%$ lower education costs and a $25 \%$ increase in access to improve education related services for five thousand children between kindergarten and high school. The solutions proposed for phase II of the CIPAA-SDGs Education Program are the Education Management Platform, and the Activities Management Platform. Where current educational programs outside of this proposal might not reliably maximize impact on the educational dimension to well-being, this program aims to define an objective measure for that wellbeing, in order to demonstrate the capacity to significantly increase that well-being.

\subsection{Education Solution Implementation in the Collective Intelligence Based Program to Accelerate Achievement of the Sustainable Development Goals (CIPAA-SDGs)}

As a conceptual example of using a value chain based on collectively intelligent cooperation to improve educational outcomes with these two platforms, assume schools are currently trying to improve educational outcomes. But assume that the government bodies regulating the disbursement of education funding generally compensate education providers according to outcomes defined in terms of specific services provided rather than general outcomes achieved, so that while individual interventions might have been proven effective in randomized trials, no 
systematic effort is made to determine whether the combination of interventions reliably achieve the optimal overall outcomes for education. Virtually all interventions are provided in isolation, and though educational is a huge industry, virtually all private and public funding processes are confined to compensating according to these non-holistic practices. Without a mechanism to maximize educational outcomes per dollar, failures in the education system are free to spiral out of control, potentially leaving part of the population without a useful education. In this sense the educational value chain is broken. But assume that with the combination of an improved Education Management Solution and an improved Activities Management Solution there is the potential to reduce costs for 5000 children by $25 \%$ through some of the means described to follow, while increasing access to improved education and activities by $25 \%$. Scaled nationally in the US, this creates the potential to help the proposed Education Freedom Scholarships (EFS) to impact up to $50 \%$ more children. Part of the President's budget request for the U.S. Department of Education for the 2021 fiscal year that he released on February 10, 2020, the EFS scholarships, which aim to "provide up to $\$ 5$ billion in additional education funding to help more than 1 million students across the country find their education fit", would then be able to impact additional 500,000 students.

To fix this broken value chain in developed countries like the US that offer charter school education vouchers, the program might define a series of projects. The first project might acquire a property at which a group of local parents of approximately twenty children combined can homeschool their children. In order to reduce this overhead, one family might reside in that property and pay rent as caretaker. To guarantee demand the project might offer parents a $50 \%$ discount for the first year in return for signing up to rent that property for the next 5 years. The second project will be to create an Education Management Solution, an Activities Management Solution, and a device through which teachers and students might interact with these platforms (the "lappad"). These software platforms might enable parents of twenty or so children to self-organize into a virtual charter school using a hybrid homeschooling model in which each group of parents use space they have in their homes already (potentially multiple rooms in multiple homes), or raise funding between them to rent or buy common facilities, and to cooperate to homeschool and to engage in other activities, or cooperate to hire teachers and activities instructors, and to take turns assisting those teachers and activities instructors, according to processes of cooperation defined by the Education Management Solution platform, and by the Activities Management Solution platform. The Education Management Solution will enable schools to reduce costs by coordinating the inclusion of children and parents into cooperating programs such as the "Lappad" affiliate marketing program that will register children into the Education Management Platform, and that will enable the parents of each of those children to register up to one hundred people in their community and personal network, so that this network might be used for marketing purposes, as well as for registration in the CIPAA-SDGs healthcare, employment, renewable energy, and other programs. Revenue from this affiliate marketing network, as well as subsidies from the other CIPAA-SDGs programs in return for registration of these potential participants, might be used to pay for or offset the cost of purchasing a lappad for each student. The lappad is a hybrid educational computer/e-reader that is each child's interface to the Education and Activities Management Solutions. And the heart of the lappad is a module transferrable between the child's lappad, their cell phone and/or home computer if they have one, or other devices they might have, to combine all of them into a safer personal communication and computing platform that can be used to engage the child with a far larger range of educational and other activities. Whether using GPS to direct children and parents to new activities and educational experiences, or connecting to sensors that taste food the child has prepared in cooking class, that measure the chemical composition of compounds from the child's chemistry experiments, or enabling parents to track the last known movements of their 
children in case children are lost, this ubiquitous personal communication and computing platform will also automate cooperation where necessary to reduce the cost of engaging in those activities. As an example, the lappad might be used to administer tests, to disseminate reading material, to schedule events and activities, and for interaction with specialist teachers in remote virtual classrooms. The Education Management Solution might facilitate those experts in creating lesson plans that tie together the specific teachings the set of students have received in other subjects, to maximize the degree to which each student integrates the subject matter in a way that serves their goals, as opposed to memorizing it for purposes that are not relevant for them. Through facilitating involvement in an individually customized set of such enriched educational activities, this program aims to create an education experience on par with that of the best private schools. In facilitating this enriched experience, the lappad has the potential to have a revolutionary impact on the problem of poor student engagement and poor student outcomes in schools. The Education Management Solution might also enable schools to create an endowment by coordinating the creation of an alumni network as students graduate from the program and as new students join it. This program might accommodate perhaps two hundred and fifty groups of parents of approximately twenty children per group, totaling five thousand children.

The third project will be to create or rent shared event, transportation, or specialized activity facilities for multiple groups. Whether these include a gymnasium, tennis court, soccer field, a hobby farm, or anything else might be decided according to the interests and preferences of the parents and students. The fourth project will be a local food services business to make healthy meals for every day and for special events. And the fifth project will be to procure fresh local foods from local growers for the same five year period. Each of these businesses cooperate though agreeing to buy key products or services from another business in the value chain, in return for another business in the chain agreeing to buy from them.
In the developing world the challenges to education might be very different. Parents might generally lack the economic flexibility or education that might allow them to homeschool. And in impoverished areas, the school fees that parents can afford demand a certain economy of scale, without which it is difficult to pay teachers or to pay for the school facilities. This limited budget leaves little room for educational enrichment. The solution is to find synergies that reduce costs and increase revenue. To fix the broken educational value chain in the developing world in doing so, might require defining a different series of projects. The first project might be to again to acquire a property at which a group of local parents of whatever number of children proves to be economic, can school their children.

This group should not be so large however as to require children to be put at risk by travelling outside of their communities. In order to reduce the overhead, one family might again reside in that property and pay rent as caretaker. To guarantee demand the project might offer parents a 50\% discount in school fees for the first year in return for signing up to attend that school for the next 5 years. This discount will be refunded at the end of the five years. The second project might be to create an Education Management Solution, and an Activities Management Solution. These software platforms will enable educators to selforganize into a virtual school network that will hire teachers and activities instructors, according to processes of cooperation defined by the Education Management Solution platform, and by the Activities Management Solution platform. This program will accommodate perhaps five thousand children. The Education Management Solution will enable schools to reduce costs by coordinating the inclusion of children and parents into cooperating programs such as the "Earn an Afripad" affiliate marketing program that will register children into the Education Management Platform, and that will enable the parents of each of those children to register up to one hundred people in their community and personal network, so that this network might be used for marketing purposes, as well as for registration in the CIPAA- 
SDGs healthcare, employment, renewable energy, and other programs. Revenue from this affiliate marketing network, as well as subsidies from the other CIPAA-SDGs programs in return for registration of these potential participants, will be used to pay for or offset the cost of purchasing an Afripad for each student. The Afripad is a hybrid educational computer/e-reader that is intended to be about the cost of books for one year. When combined with free or low-cost e-textbooks, the Afripad has the potential to have a revolutionary impact on the problem of lack of availability of learning materials in African schools. The Education Management Solution might also enable schools to increase revenues by coordinating the inclusion of the school in the CIPAA-SDGs agriculture program so that the school land can be used for agriculture, and that agricultural produce sold in local value chains.

The third project might be to create or rent shared event, transportation, or specialized activity facilities for multiple groups. These include a gymnasium, tennis court, soccer field, a hobby farm, or other, according to the interests and preferences of the parents and students. The fourth project might be a local food services business to make healthy meals for every day and for special events. And the fifth project will be to procure fresh local foods from local growers for the same five year period. Each of these businesses cooperate though agreeing to buy key products or services from another business in the value chain, in return for another business in the chain agreeing to buy from them.

Each of these projects will be profitable, and so can be funded through private investment. In the developing world, an additional opportunity for cooperation comes in finding donors or government programs who are already committed to funding such an increase in educational outcomes. Rather than these donors or governments having to pay $100 \%$ of the cost of funding new initiatives in order to improve educational outcomes, they could be offered the opportunity to cooperate with this program by providing a much smaller amount of funding merely to incentivize private investment. Having only to incentize private investment rather than funding an entire project might allow donors or governments to fund only perhaps $20 \%$ of a program, thereby multiplying their impact per educational dollar by 5 times.

Furthermore, new businesses are at best only likely to succeed $10 \%$ of the time. So funding new businesses as a way of achieving any impact such as improving educational outcomes is only likely to be successful $10 \%$ of the time. However using a "pay for success" approach in which the donor or sponsoring government pays that $20 \%$ through impact bonds that commit it to reimbursing each project only AFTER it achieves the targeted impact on outcomes, the probability of project success for the donor might increase from that $10 \%$ to near $100 \%$ per donor dollar. The businesses still won't be successful in achieving their targeted outcomes $100 \%$ of the time, but because the donor or government only pays when they are, that government will be $100 \%$ successful in its targeted outcomes per dollar spent. In going from $10 \%$ success in achieving outcomes to $100 \%$ success per dollar spent, the donor or government will multiply the impact of their funding by a further $10 \mathrm{X}$. The total increase in impact per dollar for the donor in cooperating with this program is then up to 5 times multiplied by 10 times, or 50 times.

Once successfully piloted, a value chain can potentially be replicated with private investment in a number of other locations. Assume that pilot was replicated in 15 different places. Then in this example of deploying just a few of the many potential patterns of cooperation, the result is a total increase in impact per donor dollar of up to $5 \mathrm{X}$, multiplied by $10 \mathrm{X}$, multiplied by $15 \mathrm{X}$, or 750X. This is an amazing multiplication of impact. And it doesn't require any more funding. All it requires is systematically leveraging cooperation.

In addition, rather than just allowing the donor or government to fund these impact bonds through grants of cash, the program can also enable governments to fund these impact bonds through grants of tax credits. And where the number of 
individuals whose educational outcomes can be impacted with a given amount of cash grants is finite, the number of individuals whose educational outcomes can be impacted with tax credits is as great as the capacity of the market to absorb educational impact.

\section{Conclusions}

According to a 2017 report from the U.S. Department of Agriculture, the average cost of raising a child from birth through age 17 is $\$ 233,610$ USD. Cooperating to reduce that by even half is enough to give a child an income of $\$ 20,000$ per year for five years after graduation from high school or university, so they might have the luxury of choosing a career that makes best use of their gifts. In addition, some reports suggest that the average total cost of a university education is from $\$ 101,060$ to $\$ 203,600$ USD.

- Average Total Cost of Public Colleges Per Year: $\$ 25,290$ (in-state) $\$ 40,940$ (out-ofstate)

\section{Acknowledgements.}

Thanks to Julian Talev for countless discussions on the topic.

\section{References}

1 Conference: The Relationship Between Collective Intelligence and One Model of General Collective Intelligence, Andy E. Williams, Computational Collective Intelligence, 11th International Conference, ICCCI 2019, Hendaye, France, September 4-6, 2019, Proceedings, Part II, Pages 589600

2 Pre-print: Williams, A. E. (2020, December 2). General Collective Intelligence vs the Innate Collective Intelligence Factor. https://doi.org/10.31730/osf.io/kp3x8

3 Pre-print: Williams, A. E. (2020, May 15). Use of Human-Centric Functional Modelling to Maximize Convergence in
- Average Total Cost of Private Colleges Per Year: $\$ 50,900$

Asssuming GCI can achieve the same level of potential impact as predicted in kindergarten to grade twelve education, then a GCI based university program might have the capacity to save $50 \%$ of these costs. The proposal for GCI based post-secondary educational programs is currently being developed so that university students might enjoy the same potential benefits of significantly increased outcomes per educational program dollar. Leveraging GCI to increase the quality of university education while reducing cost and also helping to ensure there is a job waiting at the end, has the potential to save another $\$ 50,000$ to $\$ 100,000$ USD, to perhaps give a child a down payment for a home of their own upon graduation, in addition to giving them a few years to find an optimal career. This head start is potentially massive.

Integrative

Research.

https://doi.org/10.31730/osf.io/jv6h8

4 Pre-print: Andy E. Williams, Discovering and Implementing Self-Sustaining Networks of Cooperation with General Collective Intelligence, working draft (2020)

5 Journal article: Woolley, Anita Williams; Chabris, Christopher F.; Pentland, Alex; Hashmi, Nada; Malone, Thomas W. (29 October 2010). "Evidence for a Collective Intelligence Factor in the Performance of Human Groups". Science. 330 (6004): 686688. Bibcode:2010Sci...330..686W. doi:10.1126/science.1193147. PMID 20929725. S2CID 74579.

6 Journal article: Krafft, P.M. (2019), A Simple Computational Theory of General Collective Intelligence. Top Cogn Sci, 11: 374-392.

https://doi.org/10.1111/tops.12341.

7 Book: Malone, T. W. (2018). Superminds: The surprising power of people and computers thinking together.

8 Pre-print: Williams, A. E. (n.d.). Individualization of Products and Services 
with Artificial General Intelligence and General Collective Intelligence. Retrieved from osf.io/preprints/africarxiv/gd5mt

9 Working Draft: Andy E. Williams, The Discrete Distributed Work Management Methodology, 2020.

10 Pre-print: Williams, A. E. (2020, April 30). The Collective Intelligence based Program to Accelerate Achievement of the Sustainable Development Goals as a Case Study for Collectively Intelligent Program Design.

https://doi.org/10.31235/osf.io/r2dxq

11 Pre-print: Williams, A. E. (2020, April 16). A Human-Centric Functional Modeling Framework for Defining and Comparing Models of Consciousness and Cognition. https://doi.org/10.31234/osf.io/94gw3 